Behaviour of Electrons in Atoms

Structure, Spectra, and Photochemistry of Atoms. By Dr. Robin M. Hochstrasser. (The General Chemistry Monograph Series.) Pp. xii +162. (New York and Amsterdam: W. A. Benjamin, Inc., 1964.) 4.50 dollars.

A STUDENT of chemistry noeds to know, at the very start of a university career, something about atomic structure and atomic spectra. However, because the interactions between electrons are complex and intricate, it is extremely difficult to give an account which is both honest and at the same time easily intelligible to the newcomer. Moreover, it is to be hoped that such an elementary description will not only be intelligible, but will also give the student some feeling and understanding for the factors that govern the energy and other properties of atomic systems.

Prof. Hochstrasser has tackled this problem in a new and interesting little book of 162 pages. The presentation is qualitative in character and does not attempt to derive mathematically the various expressions that are presented in the text. The figures are extremely good, and the use that has been made of colour in the energy level diagrams adds greatly to their intelligibility. The material is well set out and clearly written, and the summaries at the end of each chapter are most helpful. There are also some good problems.

Most of the book is very successful, but it is somewhat doubtful whether the section in the neighbourhood of page 50 will give the young student any real feeling for orbitals and the corresponding probabilities. The same is also true in respect of the energies of atomic systems, which are dealt with in Chapter 4. However, in this chapter some features are brought out more successfully than elsewhere; Fig. 4-5 is a helpful one. With regard to energies, Fig. 5.2 is wrong because all the levels for the lithium atom should be below those for the hydrogen atom which have the same principal quantum number. In Table 6-1 it would have been helpful if an energy or frequency scale had been given. Chapter 7 is very interesting, but it is doubtful whether Chapter 9 was worth including.

The author started by linking his description closely with experimental data. In the middle of the book he unfortunately departed from this approach to a considerable extent but returned to it strongly in the last few chapters. He can certainly be congratulated on providing a useful little book which will help new chemistry students in a subject which they frequently find difficult. However, they will ultimately need to carry their knowledge further.

J. W. LINNETT

\section{Stress Waves in Anelastic Solids}

Edited by Herbert Kolsky and William Prager. (International Union of Theoretical and Applied Mechanics Symposium, held at Brown University, Providence, R.I., April 3-5, 1963.) Pp. xi +342. (Berlin, Göttingen and Heidellerg: Springer-Verlag, 1964.) 67.50 D.M.

CTRESS wave problems are among the most important, $\$$ technologically, of the problems connected with deformable media. They may not be the centre of interest. Steady-state problems are likely to take pride of place so long as any important ones remain unsolved, and in recent years experiments at hypervelocities and questions about implosion have proved fascinating. But stress waves have never been far from the centre of interest, and during the past ten years solutions have been obtained to many wave problems in spite of theoretical and experimental difficultios.

The contents of Stress Waves in Anelastic Solids are the invited papers at a symposium attended by many, if not all, the experts in this field. That it should be solely concerned with anelastic solids and large strains emphasizes both the advanced stage of the theory for elastic materials and the inadequacy of that theory when there is a concentration of stress. Even the paper by Goldschmidt, at the end, giving reproducible data for such a difficult material as rock, is concerned with the dynamic as well as the static characteristics.

The two main problems in the field are the discovery of the laws of flow with large and variable strains, and the analysis of the wave patterns which arise. It is clear that steady advances are being made in both problems, but it is also clear from the papers on shock waves and those on plastic waves that the laws of flow yet known are only just adequate to correlate experiment and theory. An excellent review by Hopkins on strain-rate effected is followed by a paper by Hunter and Johnson, who analyse the propagation of small plastic vibrations along a bar, and show that the dispersion due to the finite cross-section is so appreciable at low frequencies that it could explain the results without introducing any strain-rate effects.

There are a number of papers analysing the structure of the wave pattern and its development with time. There are two on spherical and plane shocks, one on transient wave and fracture, and many dealing with waves of finite amplitude and their decay. The more interesting papers are distinguished by a final selection containing conclusions. There is a challenge here to theorists, as well as experimenters, to solve problems even more difficult than those connected with shock waves in aerodynamics. Even if the properties of the material are known, and symmetrical arrangements are chosen to avoid awkward reflexions at boundaries, these wave problems in solids remain confused owing to the internal discontinuities due to change of structure, and the associated dispersion and anisotropy.

Meanwhile, practical problems must be solved and theoretical calculations continue. There are some excellent mathematical papers on plastic waves, including one on anelastic ropes, and several on viscoelastic solids, which deserve a more complote discussion than can be given here. This is a collection of individual papers which gain by being published together.

G. J. KYNCH

\section{Numerical Methods}

By Dr. Ben Noble. Vol. 2: Differences, Integration and Differential Equations. (University Mathematical Texts.) Pp. viii + 157-372. (Edinburgh and London: Oliver and Boyd, Ltd.; New York: Interscience Publishers, Inc., 1964.) 12s. $6 d$.

THE first volume of Noble's book dealt with iterative methods for solving algebraic equations, programming, linear equations and matrix methods. In this second part, the relevant parts of the calculus of finite differences are expounded and then applied to the interpolation, differentiation and integration of tabular functions. Then there is a long chapter on ordinary differential equations, with accounts of iterative methods (Runge-Kutta), predictorcorrector methods (Adams-Bashforth, Milne), and deferredcorrector (central difference) methods. In the final chapter, we have a good if brief introduction to finite-difference and relaxation methods for partial differential equatione of the second order in two space variables. But the volume is no mere epitome of formal techniques; much attention is paid to the choice of appropriate practical methods, to error estimation and to the often subtle question of stability. There is in fact a surprising amount of information packed into this small book, for the author shows a happy skill in combining brevity with clarity. Fifty years ago, it was difficult to find a text on numerical analysis; to-day, the difficulty is one of choice out of a very large number, but the novice will get as good a view of the domain and its major routes from Noble's book as he can find anywhere. He will not get much direct help for further reading, but the author very wisely recommends for this purpose the good bibliography in Modern Computing Methods (H.M.S.O.). $\quad$ T. A. A. BroadBeit 